\title{
VIRTUAL ENTERPRISE FOR DATA MINING AND DECISION SUPPORT: A MODEL FOR NETWORKING ACADEMIA AND BUSINESS
}

\author{
Nada Lavrac ${ }^{l}$, Tanja Urbančic ${ }^{1,2}$, Andrej Orel $^{3}$ \\ IJ.Stefan Institute, Ljubljana, SLOVENIA (nada.lavrac@ijs.si, tanja.urbancic@ijs.si) \\ ${ }^{2}$ Nova Gorica Polytechnic, Nova Gorica, SLOVENIA \\ ${ }^{3}$ Marand Inženiring d.o.o., Ljubljana, Slovenia (andrej.orel@marand.si)
}

\begin{abstract}
This paper presents an organizational model for networking of international expert teams from academia and business in the area of data mining and decision support. The described virtual enterprise model was chosen as a basis for enabling a flexible collaboration of academic institutions and business entities which (although having different motivations for this partnership) share the main objective of promoting and selling advanced services offered by a pool of partners.
\end{abstract}

\section{INTRODUCTION}

Research and development projects as well as partnership networks, such as EU funded European Networks of Excellence, support the collaboration of international expert teams from academia, business and industry. The EU funded project "Data Mining and Decision Support for Business Competitiveness: A European Virtual Enterprise" (IST-1999-11495 project Sol-Eu-Net, http://soleunet.ijs.si), used as a case study in this paper, aims at forming a dynamic network of expert teams with long term experience in data mining (DM) (Fayyad et al., 1996) and decision support (DS) (Mallach, 1994) whose functionalities are complementary and oriented towards solving difficult practical problems. In this paper we propose a new organizational model in which the participating partners join their efforts and expertise in developing methods, problem-solving protocols and practical DM and DS solutions, aimed at increasing their visibility and success in the market.

In the Sol-Eu-Net Project, involving twelve partners from seven European countries, a virtual enterprise model (Camarinha-Matos et al., 2000a,b) was chosen as a basis for establishing dynamic links between experienced data mining and decision support experts on the one hand, and customers in need for specific solutions on the 
other. The novelty of this virtual enterprise model lies in a flexible association of academic institutions and business entities which (although having different motivations for this partnership) share the main objective of promoting and selling advanced services offered by the pool of partners.

In knowledge intensive services, like the ones offered by the Sol-Eu-Net virtual enterprise, success critically depends on recognizing partners' expertise, tools and skills as marketable knowledge assets. The virtual enterprise has to solve the problem of efficiently storing, updating, sharing, promoting and transferring knowledge. In addition to technological solutions, organizational, economic, legislative, psychological and cultural issues have to be addressed as well (McKenzie and Winkelen, 2001). Appropriate knowledge management (Smith and Farquhar, 2000) will lead to a quick recognition of a business opportunity and timely response (e.g., a business offer), so that geographic dispersion of clients and expert teams need not necessarily be a limit to successful business operations.

The paper presents a part of the experience of the Sol-Eu-Net Project. Section 2 introduces data mining and decision support as two main activity areas of Sol-EuNet. Section 3 outlines three organizational forms considered by the Sol-Eu-Net Project: a fixed association of project partners, a virtual enterprise with an exclusive marketing agent, and finally, a virtual enterprise involving several net-broker agents which is the current Sol-Eu-Net organizational model. Whereas the first two forms are hard to manage due to the heterogeneity of the Sol-Eu-Net Project partners' expectations and the complexity of intellectual property rights (IPR) issues that need to be solved, the latter allows for maximal flexibility in responding to new business opportunities. We conclude with a reflection on the Sol-Eu-Net Project experience.

\section{DATA MINING AND DECISION SUPPORT}

\subsection{The Sol-Eu-Net areas of expertise}

Data Mining (DM) (Shapiro et al., 1991, Fayyad et al., 1996, Han and Kamber, 2001) is concerned with finding patterns in data which are interesting (according to a user-defined measure of interestingness) and valid (according to a user defined measure of validity, e.g., classification accuracy). It is an interdisciplinary area involving databases, machine learning, pattern recognition, statistics, visualization, and others. Application areas include marketing, fraud detection, financial services, credit allocation, fault diagnosis, medical diagnosis and prognosis, and many others.

Decision Support (DS) (Mallach, 1994, Mallach, 2000) is concerned with developing systems aimed at helping decision makers solve problems and make decisions. DS provides a selection of data analysis, simulation, visualization and modeling techniques, and SW tools such as decision support systems (DSS), group decision support and mediation systems, expert systems, databases and data warehouses. DSS are designed to assist managers in semi-structured or unstructured decision-making processes. They support (rather than replace) managerial judgment and are aimed at improving the effectiveness (rather than efficiency) of decisions. 
Typical application areas are evaluation and selection of management scenarios (e.g., production, personnel), assessment of projects and investments, evaluation of companies and business partners, land-use planning, medical diagnosis and prognosis, and many others.

\subsection{The Sol-Eu-Net product}

The Sol-Eu-Net Project product consists of marketable and non-commercial goods. Since most of the products are developed for an individual customer, marketing of the product is very demanding. Moreover, IPR issues are hard to be resolved in advance, since every product requires the partners involved in product development and marketing to redefine IPR issues, depending on individual partner's involvement in each particular business opportunity. Effective marketing of products in a heterogeneous Sol-Eu-Net partnership of academic and business partners requires a flexible model of partner collaboration, outlined in Section 3.

Marketable products, whose potential clients are industry, businesses, academia and public institutions, include: DM and DS solutions to customer problems, consultancy and second-opinion evaluation of DM and DS solutions, a methodology for collaborative DM and DS, a methodology for exchanging and upgrading DM and DS models, data preprocessing tools for DM and DS, as well as educational programs in DM and DS.

On the other hand, non-commercial products, whose potential clients are academia and specialized DM and DS businesses, include: new methods for consensus building and combining problem solutions, new methods for integrating DM and DS with information systems, standards for exchanging results of DM and DS, research papers, and a virtual enterprise model, aimed at bridging the gap between academia/research and business/industry. Potential clients of the last product, the VE model, are also European R\&D projects, NoEs and businesses interested in entering virtual enterprise business-to-business relations.

\subsection{A partnership network in data mining and decision support}

The cutting edge of DM and DS technology is, on the one hand, emerging from academic/research institutions, and on the other hand, from business initiatives and research and development (R\&D) projects aimed at the development of innovative tools and techniques. For the last ten years, European collaboration between academia/research and business/industry has been supported by EU funded R\&D projects, as well as Networks of Excellence (NoE) providing infrastructure support.

R\&D projects and NoEs can be viewed as virtual enterprises emerging from a single business opportunity (an EU call for project proposals). Such a virtual enterprise has no permanent staff, in NoEs there is often not a single full-time paid individual. However, successful partner collaboration in R\&D projects and NoEs shows that strong motivation and well defined common goals allow individuals to successfully collaborate across organizational boundaries. 
The problem encountered by partners of R\&D projects and NoEs is that of identity and continuation. During the EU financing period (1-3 years) many joint results are achieved, much information gathered and disseminated, and many working relationships and workflows established. However, briefly after the end of the financing period, gathered information becomes inaccurate and workflows dissolve, since the established working relationships are not viewed as intellectual capital that should be further cultivated and exploited after the end of the project financing period. Technological products, on the other hand, are frequently successfully exploited by businesses/industry, provided that IPR and exploitation issues have been appropriately handled by project consortia.

The partnership model developed in the R\&D project Sol-Eu-Net aims at alleviating the problem of partnership discontinuation after the end of the EU funding period. A virtual enterprise (VE) model has been proposed to support business activities of a pool of DM and DS experts, following the VE definition as a temporary aggregation of core competencies and associated resources collaborating to address a specific situation, presumed to be business opportunity (Goranson, 1999).

\section{MODELS FOR NETWORKING ACADEMIA AND BUSINESS: PAST EXPERIENCE AND LESSONS LEARNED}

Having gathered core competencies and associated resources in DM and DS, a cluster of experts wanting to market their expertise has to choose a collaboration model enabling them to function as a business entity. This model should include protocols and standards for partner collaboration in distributed DM and DS projects, protocols for information gathering, settled legal and IPR issues, as well as business plans. The Sol-Eu-Net Project turns out to be an appropriate framework for developing a DM and DS partnership model. The considered models, listed in Table 1 , are the topic of this section, describing their advantages, shortcomings and lessons learned in terms of the principal-agent theory (Furubotn and Richter, 1997).

Table 1 - The Sol-Eu-Net partnership models

\begin{tabular}{|c|c|c|}
\hline Type & Form & Net-brokerage \\
\hline $\begin{array}{c}\text { Association of project partners } \\
\text { (Sec. 3.2) }\end{array}$ & Fixed & $\begin{array}{c}\text { Single marketing } \\
\text { agent }\end{array}$ \\
\hline $\begin{array}{c}\text { Virtual enterprise with a single marketing } \\
\text { agent (Sec. 3.3) }\end{array}$ & Flexible & Single net broker \\
\hline $\begin{array}{c}\text { Virtual enterprise with several marketing } \\
\text { agents (Sec. 3.4) }\end{array}$ & More flexible & Multiple net brokers \\
\hline
\end{tabular}

\subsection{The principal-agent theory}

To make the partnership effective and the whole project viable in the long run, partner relationships are regulated by a contract. Since some important relationships between partners cannot be formalized contractually, adequate care should be taken 
of social and communication aspects of collaboration. Establishing trust is the most important mechanism in cooperative projects, since the possibility of opportunistic behavior of partners cannot be eliminated by formal contracts. Means for trust building include regular communication, sharing of information and knowledge, and stable rules of the game.

The two main actors of the partnership network, the one investing intellectual capital (academic and business DM and DS experts, in the Sol-Eu-Net case) and the other investing financial capital (business partners and venture capitalists wanting to market partners' DM and DS expertise) establish the so-called principal-agent relationship (Furubotn and Richter, 1997). The reason for starting this relationship is the inability of the principal to attain his goals by himself. Defining the two roles, who is the principal and who is the agent, often depends on the context of a given business opportunity. Formally, the principal is the person/actor who is offering the contract. It is possible that in one business opportunity the same actor is the principal, and acts as the agent in another. An essential characteristic of the principal-agent relationship is that actions of the agent have two-fold consequences: on the level of agent's welfare, as well as on the utility of the principal.

The principal-agent theory gives answers to problems arising from informational asymmetries between partners in a business relationship. In theory, several types of asymmetric distribution of information are known: hidden characteristics, hidden action, hidden information and hidden intention.

- The problem of hidden characteristics arises ex ante, before signing the contract, and refers to the possibility that the principal does not know all the relevant characteristics of the agent or his services. This information is obtainable only ex post. This phenomenon, known as adverse selection, may result in choosing an unsuitable partner.

- Hidden action and hidden information asymmetries come to force ex post, during the principal-agent relationship. Hidden actions occur when the principal is not able to directly monitor actions of the agent. Hidden information happens when the principal is able to observe actions of the agent but cannot judge them as to their appropriateness because of the lack of specialized knowledge.

- Hidden intentions denote that the principal is aware of the opportunistic behavior of the agent but is unable to prevent it. This problem arises when the principal has undertaken irreversible investments making him dependent on the agent. Costs related to such investment are called sunk costs. In the case of hidden intentions, the principal does not know the intentions of the agent ex ante. This phenomenon, known as hold up situation, occurs when the agent exploits principal's dependency.

The danger of information asymmetry can be diminished before concluding the contract, by signaling agent's exceptional competences, screening of agent's characteristics, and agent's self-selection through contract formulation which disables his own opportunistic behavior. An alternative way of dealing with asymmetric information is not in overcoming this asymmetry, but in trying to 
harmonize the interests of the agent with the interests of the principal by setting up suitable measures (damage restitution, guarantees etc.).

\subsection{A fixed association of project partners}

About a year before the start of the Sol-Eu-Net Project, four of the present Sol-EuNet partners tried to form a fixed partner association, with one partner acting as an exclusive marketing agent. Some of the initial problems of this partnership can be characterized as meeting of two cultures, inherent to the academic and business world. Although the mission of the participating partners was to enable these two cultures to work together, one cannot deny numerous differences that have to be respected, rather than neglected, when aiming at a constructive partner cooperation.

The model with an exclusive marketing agent turned out to be very difficult to implement also due to other reasons. The problems of asymmetric distribution of information and the long lasting process of building trust were prohibitive to the success of the partnership. The tendency of establishing a fixed principal-agent relationships seemed to be of high priority to the marketing agent seeing himself as the principal, while DM and DS expert partners, with established means of selling their own services, didn't see themselves exclusively in the role of agents. The actors were at that point not aware of other possible forms of collaboration, or were not willing to establish them. Understandably, the partner investing financial capital wanted to have fixed guarantees that other actors will be at disposal for urgent tasks appearing in the market and he, as the principal, would set the rules of the game. On the other hand, due to the lack of information and trust, it was impossible for DM and DS expert partners to give away their academic freedom and already established business relationships, as well as other ongoing and future business opportunities. Having in mind these two completely justified, but incompatible views of the involved partners, it does not come as a surprise that the attempt of establishing a formalized association of partners had failed at that stage, despite long lasting and difficult negotiations.

\subsection{A virtual enterprise with an exclusive marketing agent}

The funding of the Sol-Eu-Net Project has provided a new opportunity for the collaboration of academic and business partners in the area of DM and DS. Twelve partners entered the project partnership, clear goals were stated and a detailed work plan was elaborated. Three years were defined as a time framework in which partners were given the opportunity to learn more about organizational aspects, in particular the ones characteristic for virtual enterprises.

A traditional enterprise has well defined boundaries, founded upon clear ownership and contractual relations, a portfolio of relatively stable resources, rule based processes, place localization and hierarchical chain of command. In contrast, a virtual enterprise (VE) features a loosely coupled collaborative arrangement with fuzzy boundaries. Fuzzy VE boundaries define functionally (not necessarily legally) self-standing organizational units, enacted as a specific organizational configuration when responding to a new business opportunity. A VE consists of autonomous 
business units (modules) that can be, on their side, parts of other systems. This modularization of the internal VE structure enables it to present itself in the market in a unitary form, while, at the same time, preserving the inner flexibility of organizational arrangements: a VE functions as an inwardly open and outwardly closed system.

The ability of engaging various highly specialized and complementary competencies and resources is the main competitive advantage of virtual enterprises and the main reason for their existence. Typically, VE modules are distributed, which enables it to reach its main advantage: the flexibility, i.e., the ability of the organization to dynamically adapt itself to changing demands and challenges.

Sol-Eu-Net partnership has decided to act as a virtual enterprise, but also to set up a company (called the SolEuNet Company in this text) in charge of product packaging, marketing and selling, while keeping for other VE partners the knowledge production function. By this decision, a two actor network has been created: some partners investing mostly their intellectual capital, and the partners of the company investing mostly the financial capital. In this situation the principal-agent theory warnings and problems, elaborated in Section 3.1, turned out to apply again. While some of the problems described in Section 3.2 were overcome when deciding for a virtual organization instead of a fixed partner association, other problems remained, mainly due to the different understanding of roles of each of the actors, amplified by the exclusive role of the marketing agent. Consequently, the idea has evolved towards the model of a virtual enterprise with distributed marketing by several netbrokers.

\subsection{A virtual enterprise with multiple net-brokers}

The differences in approaches to fulfilling particular tasks, different de-facto ways of partner practices and different cultural backgrounds invoked the idea of forming a VE with several marketing agents. In this model, the SolEuNet Company, called after the project name and formed within the Sol-Eu-Net Project, that was initially intended to act as an exclusive VE net-broker, is treated as one of several potential net-brokers forming a net-brokerage network.

An advantage of this solution is the distribution of marketing force, both geographically and socially: for instance, some customers are reluctant to dealing with entities without local presence, some others may prefer partners with a specific business background. With distributed marketing points there is good chance of better responding to business opportunities. Moreover, if from some reasons one of the net-brokers prefers not to respond to a given business opportunity, it can forward such a request to other interested net-brokers, pointing out the specific reasons for not responding himself.

Several net-brokers may lead to less tensions among partners, as dependencies are not so strong. They can also better accommodate to variable partner interests, offering particular arrangements acceptable for different partners. In terms of the principal-agent theory, this model has the advantage that net-brokers and other 
partners are better motivated to prevent asymmetric distribution of information. Namely, as there are no long-term guarantees from any side, the involved parties are more aware of the importance of building trust as one of the preconditions for successful future collaboration.

\section{CONCLUSIONS}

A virtual enterprise is a form of a voluntary partnership (exit can be exercised any time), performing cooperative task and problem solving based on the assumption of multilateral gain of all involved partners. Deciding for several net-brokers is in alignment with this understanding of a virtual enterprise. Namely, if a net-broker should be given the same possibility of leaving any time, having more than one netbroker makes a virtual enterprise less vulnerable. The paper discusses the Sol-EuNet Project experience and lessons learned. The described models may be of interest to other projects and networks wanting to enter long-term collaboration based on the virtual enterprise partnership model. The lessons learned, described in the principalagent theory framework, may help others finding a way of collaboration, considering some of the caveats to collaboration described in this paper.

\section{ACKNOWLEDGEMENTS}

The work reported in this paper was supported by the Slovenian Ministry of Education, Science and Sport, and the IST-1999-11495 project Data Mining and Decision Support for Business Competitiveness: A European Virtual Enterprise. We are grateful to Matjaž Maček for his consultancy concerning the principal-agent theory.

\section{REFERENCES}

1. Camarinha-Matos LM, Afsarmanesh $H$, Rabelo R. Supporting agility in virtual enterprises. Proceedings of PRO-VE 2000 - 2nd IFIP Working Conference on Infrastructures for Virtual Enterprises. Florianopolis, Brasil, 4-6 Dec. 2000a.

2. Camarinha-Matos LM, Afsarmanesh $H$, Rabelo $R$ (eds). E-Business and Virtual Enterprise: Managing Business-to-Business Cooperation. Kluwer Academic Publishers, $2000 \mathrm{~b}$.

3. Fayyad U, Piatetski-Shapiro G, Smith P, and Uthurusamy R (eds.) Advances in Knowledge Discovery and Data Mining. MIT Press, Cambridge, MA, 1996.

4. Furubotn EG, Richter R. Institutions and Economic Theoy: The Contribution of the New Institutional Economics. The University of Michigan Press, 1997.

5. Goranson HT. The Agile Virtual Enterprise: Cases, Metrics, Tools, Quorum Books, 1999.

6. Han J, Kamber M. Data Mining: Concepts and Techniques. Morgan Kaufmann, San Francisco, CA, 2001.

7. Mallach EG. Understanding Decision Support Systems and Expert Systems. Irwin, 1994.

8. Mallach EG. Decision Support and Data Warehouse Systems. McGraw-Hill, 2000.

9. McKenzie J, van Winkelen C. Exploring E-collaboration Space. Henley Knowledge Management Forum, 2001.

10. Piatetski-Shapiro G and Frawley W, (eds.) Knowledge Discovery in Databases. MIT Press, Cambridge, MA, 1991.

11. Smith RG, Farquhar A. The Road Ahead for Knowledge Management: An AI Perspective. AI Magazine, Vol. 21, No. 4, 17-40, 2000 